\title{
Coronary angiography utilization in the evaluation of a potential heart donor by age: a narrative review
}

\author{
Christine M. Johnstad ${ }^{1} \wedge$, Amy G. Fiedler ${ }^{2}$ \\ ${ }^{1}$ University of Wisconsin School of Medicine and Public Health, Madison, WI, USA; ${ }^{2}$ Division of Cardiothoracic Surgery, University of Wisconsin, \\ Madison, WI, USA \\ Contributions: (I) Conception and design: All authors; (II) Administrative support: AG Fiedler; (III) Provision of study materials or patients: None; (IV) \\ Collection and assembly of data: CM Johnstad; (V) Data analysis and interpretation: All authors; (VI) Manuscript writing: All authors; (VII) Final \\ approval of manuscript: All authors. \\ Correspondence to: Amy G. Fiedler, MD. Assistant Professor of Cardiothoracic Surgery, H4/230 CSC, 600 Highland Avenue, Madison, WI 53792,
} USA. Email: fiedler@surgery.wisc.edu.

\begin{abstract}
Heart transplant is the gold standard treatment for patients with heart failure. The limitation to providing heart transplantation to patients suffering from end stage heart disease is the stable organ supply within the United States despite increasing demand. Transplant centers across the United States have begun to expand traditional cardiac donor selection metrics previously utilized. As a result, the use of extended criteria donors, such as older donors, those with longer ischemic times, and donors considered high risk has increased. Current guidelines suggest that coronary angiography be performed when evaluating a donor above the age of 45. Angiographic guidelines for evaluation of the donor heart are based specifically on age, with little evidence based guidance surrounding the use of angiography in a younger donor with comorbidities or increased risk behavior which may lead to premature coronary artery disease. Recently, we have seen an increase in younger heart donors, many of whom have succumbed due to drug overdose with ensuing high risk behaviors. Given the increased risk nature of these donors, consideration of performing coronary angiography is determined by clinical "gestalt" of the transplant center evaluating the heart for use, which may lead to underutilization of donor organs without evidence to support the practice. Here, we review the guidelines, literature, and controversy surrounding the use of coronary angiography in evaluating donor hearts for transplantation.
\end{abstract}

Keywords: Coronary angiography; donor heart evaluation

Submitted Jul 28, 2020. Accepted for publication Nov 20, 2020.

doi: $10.21037 /$ jtd-20-2547

View this article at: http://dx.doi.org/10.21037/jtd-20-2547

\section{Introduction}

The true shortcoming of our ability to provide life-saving heart transplantation to patients with end stage heart failure is the stable organ supply despite an ever-growing wait list. In the United States, this has led to many transplant centers expanding the traditional donor criteria to include older donors and donors with Center for Disease Control and Prevention Increased Risk Behaviors (CDC-IR). In an effort to maximize the number of heart transplants while balancing outcomes scrutiny at a national level, transplant centers are faced with challenging decisions surrounding the acceptance of extended criteria donors. Appropriate donor selection and evaluation is therefore of critical importance in an attempt to ensure the best outcome for the recipient. A specific area within the guidelines for donor evaluation which currently lacks evidence is surrounding the use of coronary angiography to evaluate donor organs

\footnotetext{
$\wedge$ ORCID: 0000-0002-2768-2430.
} 
in the following populations: younger donors, donors with high risk behaviors (drug overdose, cocaine use, smoking history), and those with medical co-morbidities which may predispose to early coronary artery disease (diabetes, hypertension, obesity, metabolic syndrome).

We present the following article in accordance with the Narrative Review reporting checklist (available at http:// dx.doi.org/10.21037/jtd-20-2547).

\section{Methods}

Systematic electronic search was performed on PubMed, Cinhal and Cochrane database with no language limitations. We searched for the terms "coronary angiography", "heart donor evaluation", "donor heart", "drug overdose AND cardiac transplantation" from 2000 through June 2020. Titles and abstracts were independently screened based on inclusion and exclusion criteria. After irrelevant articles were eliminated, full-text articles were reviewed. We sought to employ an inclusion criterion that included studies and case reports which acknowledged the use of specific age cut-offs for coronary angiography use guidance. Exclusion criteria were exemplified by studies that did not address coronary angiography evaluation in the donor heart and/ or those lacking rationale for or against use of coronary angiography. Articles describing the use of coronary angiography in non-cardiac transplant patients were also excluded.

\section{Discussion}

\section{Current donor considerations for angiography}

The limited availability of donor hearts is well recognized which remains the rate limiting step to growing the volume of heart transplantation. Due to this, the transplant community has an interest in expanding what is considered the traditional donor pool by evaluating donor hearts outside of the standard criteria. The International Society for Heart and Lung Transplantation (ISHLT) has modified its criteria in order to have minimal absolute contraindications to heart transplantation, and many prior absolute contraindications are now relative (1). The seemingly infinite demand for heart donors has led to the utilization of older donors beyond the traditional acceptance criteria. Many centers arbitrarily use a donor age cutoff of $<55$ years, while others will accept donors up to 65 years, and in some instances even greater (2). Even with these efforts to expand the donor pool through the use of increasingly older donors, it has been observed that there is a large underutilization of potential organ donors, most likely in an attempt to avoid complications. Authors cite concerns over the use of extended criteria donor (ECD) hearts as long term results of recipients of these donors' remains scarce. Evidence demonstrates that ECD hearts with the potential for favorable outcomes continue to be refused and discarded (3).

With respect to older donor hearts, concerns are raised for donor transmitted CAD, resulting in increased postoperative mortality, and early development of cardiac allograft vasculopathy (CAV). These increased risks, however, may be outweighed when confronted with the alternative of a waitlist death $(2,4)$. Donor transmitted CAD is of particular concern due to its significant association with early graft dysfunction when multi-vessel disease is present $(5,6)$. ISHLT guidelines recommend that donor hearts with obstructive $\mathrm{CAD}$ in any major coronary artery be rejected (7). However, single vessel CAD does not differ in short or long-term prognosis when compared to donors without CAD (5).

On the other hand, there has been a marked increase in young donor heart availability due to the opioid epidemic. Due to the nature of the donor's death, most of these organs are considered CDC-IR. Interestingly, despite the young age of the donors, these hearts may be declined by transplant centers, despite evidence indicating that survival is not affected if appropriately screened. While little is known about the natural history of a young donor heart into a recipient, several studies have demonstrated that donors with cause of death being drug overdose tended towards increased tobacco use which is a well-documented risk factor for the development of coronary artery disease $(8,9)$. Younger, high risk donors may also have specific comorbidities, such as diabetes, hypertension, and obesity which may predispose them to early development of CAD. Additionally, in this specific population, the prevalence and use of cocaine, which is known to be a coronary vasoconstrictor, is high. Given this constellation of considerations, it can be difficult for transplant centers to determine if coronary angiography, or alternative metrics to evaluate coronary atherosclerosis such as coronary gated CT scan, or stress echocardiography should be requested when considering these donors. Challenges may also ensue if the donor hospital does not have the capacity to perform diagnostic coronary angiography or alternative coronary atherosclerosis diagnostic methods. This scenario may lead 
to a situation where the higher sequence transplant center declines an organ due to inability to perform screening angiography, and while the organ may ultimately be placed at a lower sequence, this situation is not ideal.

Current guidelines for heart donor evaluation in the United States require that all potential donor hearts be screened with two-dimensional echocardiography, whereas coronary angiography is variable and circumstantial (10). Many transplant centers in the United States follow either The Association of Organ Procurement Organizations Consensus Statement or the American College of Cardiology and American Heart Association task force guidelines. The Association of Organ Procurement Organizations Consensus Statement, supported by the Society of Critical Care Medicine and the American College of Chest Physicians recommends coronary angiography in all older donors ( $>40$ years) and in younger patients with risk factors for premature CAD. Risk factors include hypertension, diabetes, smoking history, dyslipidemia, family history of premature $\mathrm{CAD}$, and history of cocaine abuse (5-7). Alternatively, the American College of Cardiology and American Heart Association task force developed guidelines in 1987, which recommends coronary angiography for male donors ( $>45$ years) and female donors ( $>50$ years). An additional suggestion from this task force was to decrease the age threshold by 5 to 10 years in the presence of cardiac risk factors (11).

When donor coronary angiography is routinely utilized, research demonstrates that cardiac transplantation rates increase. A European study demonstrated that when angiography of donor hearts is performed and has normal findings, transplant rates are significantly higher than if their angiography was deferred (12). Another study found that by performing coronary angiography in ECD hearts, the acceptance rate of those donors was increased by $9 \%$. Often, hearts are rejected by centers due to the presence of risk factors even without objective evaluation of the organ via angiography (5).

Certain groups have advocated for more liberal if not standardized use of coronary angiography in the evaluation of donor hearts. The rationale being the likelihood of increased acceptance rates of organs which may be otherwise discarded. Evidence has indicated that in all comers, CAD is identified in one or more arteries in nearly one third of donor hearts that are thought to be "normal" (13). In the multi-visceral donor, there has been concern regarding the use of coronary angiography on renal function due to the effect of the dye load on kidney function. That said, evidence shows that the use of contrast dye in the evaluation of a heart donor has not led to impairments in donor kidney function. These findings should minimize the apprehension surrounding applying screening coronary angiography to most donor hearts (6).

Current guidelines for coronary angiography evaluation serve as recommendations for donors based on increased age and/or risk factors. As a result, transplant centers and accepting physicians are reliant upon their own personal experience and clinical judgement to determine the utility or need for coronary angiography in a variety of donors based on age, mode of death, and increased risk behaviors. The lack of recent and clearly defined guidelines for donor evaluation along the spectrum of age leads to ambiguity in the use of coronary angiography in the donor evaluation.

\section{Use of coronary angiography in the younger donor}

A case report of a patient undergoing heart transplant with a donor heart from a male in his early twenties whose cause of death was cocaine overdose, did not undergo coronary angiography evaluation pre-transplant due to the donor's young age, despite risk factors. Post-transplant, the recipient had routine screening coronary angiography to evaluate for graft vasculopathy which revealed a significant myocardial bridge. This case reveals the potential harm of not screening young donors with angiography prior to allocating the organ for transplant. Additionally, should a recipient receive a young heart with undiagnosed coronary artery disease the inability to manifest classic anginal symptoms due to denervation during transplant may leave the recipient in a particularly vulnerable position. Formal guidelines do not exist regarding the evaluation of a donor heart under the age of 45 with respect to screening coronary angiography, possibly leading to underutilization of the organ or the organ being placed at a lower sequence due to center hesitation surrounding acceptance. Current practice indicates that coronary angiography for young donors in the absence of significant risk factors has historically been largely deferred, although given the rise in the opioid epidemic, many institutions have begun to consider highrisk behaviors and history of drug abuse as indications for requesting donor coronary angiography (14).

Hauptman et al. (11) published the experience of the New England Organ Bank (NEOB) and diagnostic coronary angiography in donor evaluations from 1993 through 1997. While this manuscript was focused on the use and performance of coronary angiography and donor 
hearts in the age group between 40-65 years of age, a subanalysis was conducted of those donors less than 40 whom underwent coronary angiography in the donor evaluation process. This small cohort consisted only of 15 donors. Surprisingly, of the 15 donors less than 40 years of age, 4 of the donors were not transplanted due to concerns identified on coronary angiography that were not apparent on screening echocardiography. Albeit a small cohort, this data is intriguing, as many centers may have accepted these organs without angiography due to the age of the donor. This should give the transplant community pause, and spur further research into the utility of donor coronary angiography in the younger population.

\section{Limitations in current practice}

Some of the hesitation surrounding the universal application of coronary angiography across all donor organs may be related to availability of interventional cardiology at the hospital where the donor is being cared for. In these circumstances, there are opportunities for coronary angiography to occur even when it is not feasible at specific institutions.

If approved by the Federal Drug Administration (FDA) for use in clinical practice (pending Phase 3 results) the TransMedics Organ Care System offers a beatingheart perfusion chamber, with the capability for ex-vivo coronary angiography when pre-harvest evaluation cannot be performed (15). If coronary angiography evaluation is not performed pre-transplant there is a possibility of considerable financial consequences from retrievable costs if the donor heart is determined to be unfit for transplant $(16,17)$. Another point of consideration is in the ability to form a baseline assessment with coronary angiography. CAV, a major concern for recipients, is routinely assessed for posttransplant. Therefore, coronary angiography evaluation pre-transplant would not only offer a comparative baseline but it would also allow for coronary pathology screening.

\section{Summary}

Information to inform clinical decision making is paramount, which begs the question, should coronary angiography evaluation become standard of care pretransplantation? The cost-to-benefit ratio of this potential implementation currently remains unknown. The absence of guidelines for evaluation of young donors highlights the necessity of further studies to provide evidence-based guidance to providers. Large, multicenter evaluation of current practices in the United States related to potential donor heart coronary angiography evaluation with outcome follow-up is warranted.

\section{Acknowledgments}

Funding: None.

\section{Footnote}

Reporting Checklist: The authors have completed the Narrative Review reporting checklist. Available at http:// dx.doi.org/10.21037/jtd-20-2547

Conflicts of Interest: All authors have completed the ICMJE uniform disclosure form (available at http://dx.doi. org/10.21037/jtd-20-2547). The authors have no conflicts of interest to declare.

Etbical Statement: The authors are accountable for all aspects of the work in ensuring that questions related to the accuracy or integrity of any part of the work are appropriately investigated and resolved.

Open Access Statement: This is an Open Access article distributed in accordance with the Creative Commons Attribution-NonCommercial-NoDerivs 4.0 International License (CC BY-NC-ND 4.0), which permits the noncommercial replication and distribution of the article with the strict proviso that no changes or edits are made and the original work is properly cited (including links to both the formal publication through the relevant DOI and the license). See: https://creativecommons.org/licenses/by-nc-nd/4.0/.

\section{References}

1. Sellke FW, Ruel M, Rao V. Heart Transplantation. In: Atlas of cardiac surgical techniques. Philadelphia, PA: Elsevier; 2019. p. 546-65.

2. Kilic A, Emani S, Sai-Sudhakar CB, et al. Donor selection in heart transplantation. J Thorac Dis 2014;6:1097-104.

3. Kittleson MM, Kobashigawa JA. Cardiac Transplantation: Current Outcomes and Contemporary Controversies. JACC Heart Fail 2017;5:857-68.

4. Estevez-Loureiro R, Paniagua-Martin M, Calviño-Santos $\mathrm{R}$, et al. Prevalence of Donor-Transmitted Coronary Artery Disease and Its Influence on Heart Transplant 
Outcomes. Transplant Proc 2010;42:2987-91.

5. Ivanes F, Cantrelle C, Genet T, et al. Performing diagnostic coronary angiography to evaluate high-risk cardiac donors: A French nationwide cohort study. Int J Cardiol 2019;277:71-8.

6. Kotloff RM, Blosser S, Fulda GJ, et al. Management of the Potential Organ Donor in the ICU. Crit Care Med 2015;43:1291-325.

7. Dorent R, Gandjbakhch E, Goéminne C, et al. Assessment of potential heart donors: A statement from the French heart transplant community. Arch Cardiovasc Dis 2018;111:126-39.

8. Ising MS, Gallo M, Whited WM, et al. Changing demographics of heart donors: The impact of donor drug intoxication on posttransplant survival. Am J Transplant 2018;18:1790-8.

9. Warraich HJ, Lu D, Cobb S, et al. Trends and outcomes of cardiac transplantation from donors dying of drug intoxication. Am Heart J 2018;199:92-6.

10. Zipes DP, Libby P, Bonow RO, et al. Surgical Management of Heart Failure. In: Braunwald's heart disease: a textbook of cardiovascular medicine. Philadelphia, PA: Elsevier; 2019. p. 553-67.

11. Hauptman PJ, O'Connor KJ, Wolf RE, et al. Angiography

Cite this article as: Johnstad CM, Fiedler AG. Coronary angiography utilization in the evaluation of a potential heart donor by age: a narrative review. J Thorac Dis 2021;13(3):18641868. doi: $10.21037 /$ jtd-20-2547 of potential cardiac donors. J Am Coll Cardiol 2001;37:1252-8.

12. Zuckermann A, Auersperg K, Kaider A, et al. Coronary Angiography of Potential Cardiac Donors Increases Cardiac Transplantation Rates. Transplantation 2018;102. doi: 10.1016/j.healun.2018.01.416.

13. Guglin M, Omar H, Wright C. Underutilization of Donor Hearts: An Observational Study. Transplant Proc 2018;50:3698-704.

14. Dahiya G, Mcquade D, Alpert C. Myocardial bridging in the era of a drug epidemic: a case report addressing the need to revisit donor organ assessment. Eur Heart J Case Rep 2019;3:1-4.

15. García Sáez D, Zych B, Sabashnikov A, et al. Evaluation of the organ care system in heart transplantation with an adverse donor/recipient profile. Ann Thorac Surg 2014;98:2099-105; discussion 2105-6.

16. Anthony C, Michel J, Christofi M, et al. Ex Vivo Coronary Angiographic Evaluation of a Beating Donor Heart. Circulation 2014;130:e341-3.

17. Ghodsizad A, Bordel V, Ungerer M, et al. Ex vivo coronary angiography of a donor heart in the organ care system. Heart Surg Forum 2012;15:E161-3. 